A Method of Preparing Sections of Fossil Plants contained in Coal Balls or in other Types of Petrifaction.

The method described in this letter was devised in collaboration with Dr. R. G. Koopmans of Utrecht. A flat surface must first of all be cut or ground on the coal ball or other petrified mass parallel to the planes in which the sections are required: this surface is ground smooth, but a fine polish is not necessary. The surface is then immersed for a definite length of time in an etching solution of hydrochloric acid, the optimum concentration being found by trial. The acid dissolves away a film of the carbonate of which the mass is largely composed, and the plant substance contained in the film is left behind, standing in relief above the surface. The surface is washed carefully and dried. A solution or fluid, which on drying or hardening forms a tough film, is then poured over the surface and allowed to dry or harden. Several solutions have been used for this purpose; the best as regards consistency is the trade preparation 'Durofix,' but other solutions containing cellulose compounds may be used. When quite hard this film of cellulose-compound may be peeled off, as it has considerable tensile strength. The plant substance which was left in relief by the etching process is embedded in the film and is detached with it from the surface of the petrifaction. The film is then washed, first in the acid and then in water, dried, cleared, and finally mounted in Canada balsam between a slide and coverslip. The surface of the petrified mass is gently rubbed down on fine carborundum paper and is then ready for the removal of another section.

These sections consisting of the plant substance embedded in a film of cellulose compound are scarcely distinguishable from good petrological slices cut from the petrified mass direct, and are usually much superior. The thickness of the plant section depends on the length of exposure to the acid bath, and may be of a very small order if so desired. The sections are of uniform thickness and are almost unbreakable. From the botanical point of view, the main advantage of the new method is that almost continuous serial sections may be prepared, since the old method of cutting petrological slices allowed at the most three sections to five millimetres of material. The sizes of the films are limited only by the size of the petrified mass from which they are made. When a petrifaction contains several different objects, it may be convenient to cut up the films with scissors and mount the parts separately. The cost of making a film is negligible compared with the cost of preparing a petrological slice, which involves in its production a considerable amount of hand labour. With silicified material hydrofluoric acid must be used instead of hydrochloric.

No attempt has yet been made to apply the method to plants preserved in pyrites, but here again a suitable acid must be chosen to dissolve the pyrite.

Department of Botany,

University of Birmingham.

\section{Condition of Radium Salts after Storage in Sealed Glass Tubes.}

IT has recently been found necessary in the Grovernment Laboratory to open and subdivide two tubes containing radium salts which were sealed by us in 1921 , and it is thought that the experience gained in opening them may be of interest to others working with radium preparations of high grade.
The tubes contained 171.8 and 54.9 milligrams of radium element as 92 per cent chloride and 50 per cent bromide respectively. These salts had been dried at $240^{\circ} \mathrm{C}$. for two hours before they were sealed into thin glass tubes, each furnished with a small piece of fine platinum wire fused into it.

To open the tubes a method believed to have been devised by the late Mr. Harrison Glew was used. Each was placed in a clean lead tube such as is used to contain tooth-paste, and the lead folded down on the glass. By applying pressure with a screw jaw spanner the radium tube was then cracked, whereupon the lead tube was slit with scissors and the radium salt washed out.

We had been led by other workers to expect that there would be a considerable gas pressure in the tubes, arising from the decomposition of water, but no sign of this was observed, as, for example, by distension of the tube. The glass of the tubes, although strongly coloured, showed no apparent disintegration or deterioration from the action of the rays. It may be concluded, therefore, that considerable quantities of high grade radium salts, sealed in glass tubes, ean safely be kept unopened for periods of at least seven years, provided the drying has been thorough.

The effect of the action of the radium on the labels and packing may be mentioned. Thus on evaporating the filtered radium solutions a gummy organic impurity was observed, apparently produced under the action of the rays from the paper labels on the tubes and from cotton-wool packing. The cotton had completely disintegrated, some traces only adhering to the tubes. It has been observed in this laboratory that when cellulose is exposed to $\beta$ and $\gamma$ rays in presence of air, it is chemically altered, the proportion of material soluble in 3 per cent caustic soda solution notably increasing. It would thus seem advisable to avoid attaching any label or other foreign material directly to the glass of tubes containing radium, and to store the tubes out of contact with organic matter.

Two further points may be mentioned as regards recovery. To purify the contaminated radium, it was ignited at a faint red heat in silica dishes. After this treatment the dishes were found to be distinctly radioactive, and upon treatment with hydrofluoric acid four dishes yielded 0.3 milligram of radium which had not been removed by means of hydrochloric or hydrobromic acid.

Finally, it may be mentioned that more than 98.5 per cent of the radium salt, after it had been kept in a sealed glass tube for seven years, was still in a state soluble in dilute acid. A. G. Francrs.

$$
\begin{aligned}
& \text { Clement's Inn Passage, } \\
& \text { Strand, W.C.2, } \\
& \text { Sept. } 24 .
\end{aligned}
$$

The Crystal Structure of Solid Methane.

$I_{N}$ view of the recent controversy concerning the structure of the methane molecule, it is proposed to state briefly the results of an X-ray "powder" investigation of solid methane at a temperature intermediate between that of liquid air and liquid hydrogen.

Only one modification, a cubic one, was found, and the observed spacings indicated a structure based upon a face-centred lattice, the unit cell having an edge $6.35 \mathrm{~A}$. and containing 4 molecules of methane. (This gives, for solid methane, a density of 0.413 gm./c.c., while that of liquid methane at its boilingpoint, $-164^{\circ} \mathrm{C}$., has been given as $0.415 \mathrm{gm}$./c.c.)

There are no abnormal spacings except those characteristic of a face-centred lattice, hence the

No. 3076, VoL. 122] 